\title{
Calpain and spectrin breakdown products as potential biomarkers in tuberculous pleural effusion
}

\author{
Ji Young Hong ${ }^{1,2,3}$, So Yeong Park ${ }^{1,3}$, Youngmi Kim ${ }^{3}$, Chang Youl Lee ${ }^{1,2}$, Myung Goo Lee ${ }^{1,2}$ \\ ${ }^{1}$ Division of Pulmonary, Allergy and Critical Care Medicine, Department of Internal Medicine, Chuncheon Sacred Heart Hospital, Hallym \\ University Medical Center, Chuncheon, Gangwon-do, Republic of Korea; ${ }^{2}$ Lung Research Institute, ${ }^{3}$ Institute of New Frontier Research, Hallym \\ University College of Medicine, Chuncheon, Gangwon-do, Republic of Korea \\ Contributions: (I) Conception and design: JY Hong; (II) Administrative support: SY Park, Y Kim; (III) Provision of study materials or patients: CY \\ Lee, MG Lee; (IV) Collection and assembly of data: CY Lee, MG Lee; (V) Data analysis and interpretation: JY Hong, MG Lee; (VI) Manuscript \\ writing: All authors; (VII) Final approval of manuscript: All authors. \\ Correspondence to: Myung Goo Lee, MD, PhD. Professor, Division of Pulmonary and Critical Care Medicine, Department of Medicine, Chuncheon \\ Sacred Heart Hospital, Hallym University Medical Center, 77, Sakju-ro, Chuncheon-si, Gangwon-do 200-704, Republic of Korea. \\ Email: mglee@hallym.or.kr.
}

\begin{abstract}
Background: Early diagnosis of tuberculous pleural effusion (TPE) remains difficult. Calpain is a family of calcium-dependent endopeptidase that plays an important role in extracellular matrix (ECM) remodeling and collagen synthesis. The aim of this study was to explore the diagnostic value of pleural fluid angiotensinconverting enzyme (ACE), calpain-1, spectrin breakdown products (SBDP), and matrix metalloproteinase-1 (MMP-1) in TPE and malignant pleural effusion (MPE).

Methods: The study included 47 patients with TPE, 28 patients with MPE, and 10 patients with transudate of non-tuberculous and non-malignant origin as controls. Calpain-1, ACE, SBDP, and MMP-1 levels in pleural fluid were measured by the ELISA method.

Results: ACE, calpain-1, SBDP, and MMP-1 levels were higher in TPE than MPE and transudate (all, $\mathrm{P}<0.05$ ). On multivariate logistic regression analysis, adenosine deaminase (ADA) $\geq 40 \mathrm{IU} / \mathrm{mL}$, calpain- 1 $\geq 787 \mathrm{ng} / \mathrm{mL}$, and SBDP $\geq 2.745 \mathrm{ng} / \mathrm{mL}$ were independent factors associated with TPE. The predicted probability of TPE based on these three predictors had an area under the receiver operating characteristic (ROC) curve of 0.985 , with $97.9 \%$ sensitivity and $86.6 \%$ specificity under a cut-off value of 0.326 . In patients with TPE, residual pulmonary thickening (RPT) was associated with significantly higher calpain-1, SBDP, and MMP-1 levels (all, $\mathrm{P}<0.05$ ) versus cases without RPT.

Conclusions: Our results suggest that the overproduction of calpain-1 and SBDP is associated with pleural fibrosis in tuberculous pleurisy. While ADA is a conventional marker for diagnostic TPE, the simultaneous measurement of calpain-1 and SBDP 1 in pleural fluid may improve the diagnostic efficacy.
\end{abstract}

Keywords: Tuberculous pleural effusion (TPE); calpain; biomarker

Submitted Aug 07, 2017. Accepted for publication Apr 10, 2018.

doi: $10.21037 /$ jtd.2018.04.85

View this article at: http://dx.doi.org/10.21037/jtd.2018.04.85

\section{Introduction}

Tuberculosis (TB) remains the leading infectious cause of mortality and morbidity. According to estimates by the World Health Organization, there were 10.4 million new cases, and 1.8 million deaths, in 2015 worldwide. In Korea, 30,000 incident cases and 2,300 deaths are reported annually.

Tuberculous pleurisy is the most common form of extrapulmonary TB, accounting for $5 \%$ of all TB cases (1). Early diagnosis of tuberculous pleural effusion (TPE) is difficult. Exudative lymphodominant effusion, determined by routine analysis of $\mathrm{PE}$, is common in etiologies other 
than TPE. Moreover, pulmonary parenchymal TB is found in only $20-50 \%$ of TPE cases, and only $52 \%$ of TPE cases have a positive mycobacterial sputum culture (2). The sensitivity and specificity of adenosine deaminase (ADA), a well-known marker, are high, but these can also be increased in other diseases, such as rheumatoid arthritis, empyema, mesothelioma, chronic lymphocytic leukemia, and mycoplasma pneumonia. Therefore, highly sensitive and specific biomarkers to differentiate TPE from effusions caused by other etiologies are urgently needed.

Pleural fibrosis, a common complication of TPE, occurs in $50 \%$ of patients with TPE (3). Because excessive deposition of extracellular matrix (ECM) after an inflammatory condition leads to lung entrapment and chronic respiratory failure in tuberculous pleurisy, the mechanism of pleural fibrosis requires clarification. Calpain is a family of calcium-dependent endopeptidases that plays an important role in ECM remodeling, pulmonary vascular remodeling, and bleomycin-induced pulmonary fibrosis $(4,5)$. Calpain-mediated degradation of spectrin results in the formation of spectrin breakdown products (SBDP). Yang et al. reported that angiotensin-converting enzyme (ACE)-angiotensin (Ang) II-activated calpain mediates tuberculous pleural fibrosis (6). Chen et al. showed that matrix metalloproteinase-1 (MMP-1), the main protease for tissue destruction and remodeling, is significantly associated with the degree of residual pleural fibrosis in TB pleurisy (7).

The aim of this study was to investigate the diagnostic implications of fibrosis-related markers (ACE, calpain, SBDP, and MMP-1) as novel biomarkers in TPE compared with malignant pleural effusion (MPE) or transudate. In addition, we studied the relationship between fibrosisrelated markers in pleural fluid and residual pleural thickening in TPE.

\section{Methods}

\section{Patient enrollment}

This prospective study was conducted at Chuncheon Sacred Heart Hospital in Korea from January 2010 to June 2016. Approval for the study was granted by the hospital's Research Ethics Committee (institutional review board number: 2012-27). During the study period, 164 patients aged $>18$ years required thoracentesis. Among them, four subjects were defined as having hemothorax and forty-nine subjects were defined as having parapneumonic effusion. Twenty-six subjects had PE of mixed etiology. Patients with hemothorax, parapneumonic effusion, and effusion of uncertain etiology were excluded. Finally, 85 patients were enrolled.

\section{Data collection}

Clinical data, including age, sex, comorbid diseases and radiologic and laboratory findings, were recorded at enrollment. All PE samples were collected during routine thoracentesis. Total cell differential counts, total protein, glucose, lactate dehydrogenase $(\mathrm{LDH})$, protein and albumin levels, $\mathrm{pH}$, cytology, and cultures of bacteria and mycobacterium were obtained.

PEs were divided into 75 exudative PEs and 10 transudative PEs according to Light's criteria (8). The exudative PEs were further divided into 47 tuberculous effusions and 28 malignant effusions.

Tuberculous effusion was defined by pleural fluid that was culture-positive for M. tuberculosis or histologically confirmed tuberculous infection by pleural biopsy. Probable tuberculous pleurisy was defined based on fulfilment of one of the following criteria: (I) a definite good response to anti-TB medication without other possible causes of $\mathrm{PE}$ or (II) M. tuberculosis-positive sputum. Malignant effusion was defined based on fulfilment of both of the following criteria: (I) M. tuberculosis-negative pleural fluid and (II) a histopathological or cytological diagnosis of tumors.

Residual pulmonary thickening (RPT) was defined as a pleural thickness $\geq 10 \mathrm{~mm}$ on the lateral-inferior portion of a posteroanterior chest roentgenogram, in accordance with a previous study $(3,9)$.

\section{Measurement of PE biomarkers}

Ten milliliters of pleural fluid were collected in sterile tubes from all patients via thoracentesis. The pleural fluid was centrifuged at $3,000 \mathrm{~g}$ for 10 minutes and the supernatant was frozen at $-80^{\circ} \mathrm{C}$ for analysis.

An enzyme-linked immunosorbent assay (ELISA) was used to measure ACE (R\&D Systems, Minneapolis, MN, USA), calpain-1 (Elabscience Biotechnology Co., Ltd., Wuhan, China), MMP-1 (Abcam, Cambridge, UK), and SBDP145 (Wuhan Fine Biological Technology Co., Ltd, Wuhan, China). The assays were performed according to the manufacturers' instructions. ADA activity was determined using a commercial assay kit (Toyobo Co., Ltd., Osaka, Japan). 


\section{Statistical analysis}

Intergroup differences were analyzed by the MannWhitney $U$ test for numerical variables and by the chisquared test for categorical variables. The ELISA results of each biomarker were transformed from continuous variables to dichotomous variables based on the Youden index of the receiver operating characteristic (ROC) curve.

Multivariate logistic regression analysis was used to identify independent factors for TPE, and to predict the probability of TPE for each patient based on the model equation.

ROC curve analysis was applied to determine the optimal cut-off value for the logistic regression analysis. Statistical significance was set at $\mathrm{P}<0.05$. All analyses were performed using GraphPad (ver. 5.1; GraphPad Software, Inc., La Jolla, CA, USA) and SPSS (ver. 20.0; SPSS Inc., Chicago, IL, USA) statistical software.

\section{Results}

In total, 85 patients were enrolled, including 47 with TPE, 28 with MPE, and 10 with transudate.

TPE was diagnosed by a positive culture of PE in 11 patients, positive sputum culture in 5 patients, typical pleura histology in 11 patients and positive nucleic acid amplification test of PE in three patients. Seventeen patients responded positively to anti-tuberculous medications without other possible causes of PE. The median age of the enrolled patients was 62.1 years, and 35 out of TPE 47 patients were male.

In the MPE group, all cases were histologically confirmed by cytology or pathology, including 21 cases of primary cancer, one case each of thymic cancer, plasmacytoma, renal cell carcinoma, colon cancer, breast cancer, endometrial cancer, and malignant mesothelioma.

There were more male subjects in the TPE group than in the non-TPE group (74.5\% vs. 52.6\%; Table 1). Neither the presence of comorbid disease nor age were significantly different between the two groups. Comorbid disease was more prevalent in patients with transudate versus those with non-transudate (100\% vs. $48 \%)$. The proportion of bilateral effusion and hemithorax of more than two thirds was significantly higher among patients with transudate. As expected, the ADA levels of PE were higher in the TPE group than in the non-TPE group. The lymphocyte to neutrophil $(\mathrm{L} / \mathrm{N})$ ratio of pleural effusion was analyzed. The median $\mathrm{L} / \mathrm{N}$ ratio of pleural effusion was significantly higher for TPE than non-TPE group (TPE: 4.0, non-TPE: $2.3, \mathrm{P}=0.002)$. This result was consistent with the previous study that lymphocyte/neutrophil ratios is an useful test in the diagnosis tuberculous pleuritis (10).

The PE biomarker results are shown in Figure 1. ACE, calpain-1, SBDP, and MMP-1 levels were highest in the TPE group. The median ACE level of TPE $(53.38 \mathrm{ng} / \mathrm{mL})$ was significantly higher than that of MPE $(34.73 \mathrm{ng} / \mathrm{mL}$, $\mathrm{P}<0.001)$ and transudate $(25.37 \mathrm{ng} / \mathrm{mL}, \mathrm{P}<0.001)$. The median calpain-1 level of TPE $(922.3 \mathrm{ng} / \mathrm{mL})$ was significantly higher than that of MPE $(797.8 \mathrm{ng} / \mathrm{mL}$, $\mathrm{P}=0.010)$ and transudate $(835.3 \mathrm{ng} / \mathrm{mL}, \mathrm{P}=0.041)$. The median SBDP level of TPE $(6.87 \mathrm{ng} / \mathrm{mL})$ was significantly higher than that of MPE $(1.79 \mathrm{ng} / \mathrm{mL}, \mathrm{P}<0.001)$ and transudate $(0.01 \mathrm{ng} / \mathrm{mL}, \mathrm{P}<0.001)$. The median MMP1 level of TPE $(14,970 \mathrm{pg} / \mathrm{mL})$ was significantly higher than that of MPE $(6,087 \mathrm{pg} / \mathrm{mL}, \mathrm{P}=0.002)$ and transudate (735.6 pg/mL, $\mathrm{P}<0.001)$. Among the PE biomarkers, SBDP had the highest area under the ROC curve (AUC) (0.831), followed by ACE (0.803), MMP-1 (0.786), and calpain-1 (0.716) (Figure 2A). With a cut-off value of $47.16 \mathrm{ng} / \mathrm{mL}$, pleural ACE had $55.3 \%$ sensitivity and $94.7 \%$ specificity, whereas pleural calpain-1, with a cut-off value of $787 \mathrm{ng} / \mathrm{mL}$, had a sensitivity and specificity of $95.7 \%$ and $47.3 \%$, respectively. With a cut-off value of $2.745 \mathrm{ng} / \mathrm{mL}$, pleural SBDP had $87.2 \%$ sensitivity and $78.9 \%$ specificity. For pleural MMP-1, 74.4\% sensitivity and $65.7 \%$ specificity were observed with a cut-off value of $7,229 \mathrm{pg} / \mathrm{mL}$.

Multivariate logistic regression analysis using dichotomous variables identified by the cut-off value of the ROC curve was performed to identify predictors of TPE. $\mathrm{ADA} \geq 40 \mathrm{IU} / \mathrm{mL}$ [odds ratio (OR): 1,165.2, 95\% confidence interval (CI): 8.3-163,621.8], calpain-1 $\geq 787 \mathrm{ng} / \mathrm{mL}$ (OR: 59.6, 95\% CI: 1.3-2,753.9), and SBDP $\geq 7,229 \mathrm{pg} / \mathrm{mL}$ (OR: 38.3, 95\% CI: 1.3-1,112.4) were independent predictors (Table 2). By using three independent predictors, the predicted probability of TPE was calculated according to the model equation:

Logit $($ probability $)=\operatorname{Ln}($ probability $/ 1-$ probability $)=$ $[5.361 \times($ presence of ADA at $\geq 40 \mathrm{IU} / \mathrm{mL})]+[4.120 \times$ (presence of calpain-1 $\geq 787 \mathrm{ng} / \mathrm{mL}$ ) $]+[3.682 \times$ (presence of SBDP at $\geq 2.745 \mathrm{ng} / \mathrm{mL}$ )] -7.585

If one of the factors in the formula is higher than or equal to the cut-off, the score for that factor is set to one. If the factor is smaller than the cut-off, the score is set to zero. ROC curve analysis showed that the predicted probability had an AUC of 0.985, with an optimal cut-off value of 0.326 
Table 1 Clinical characteristics of the study participants

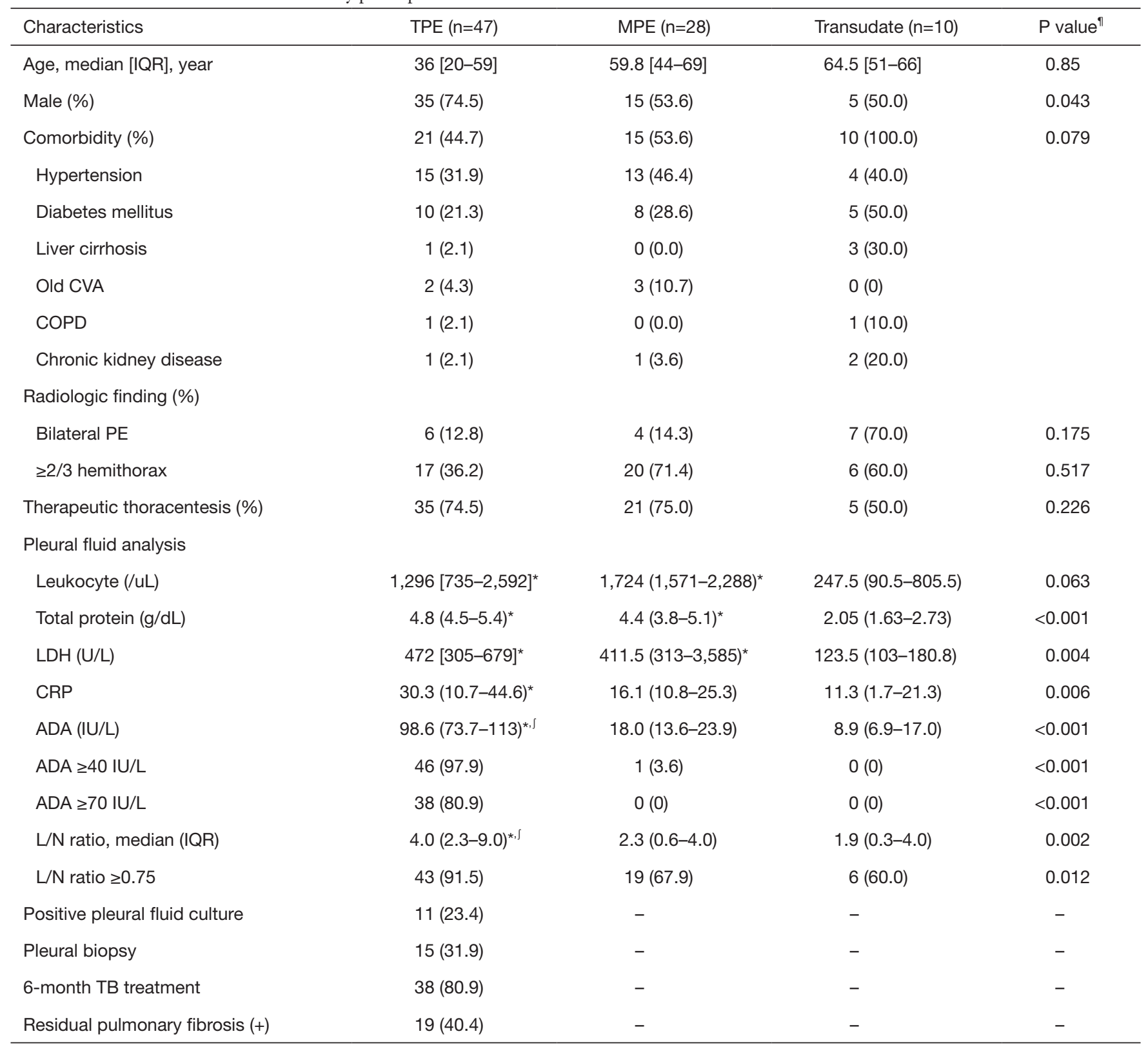

*, $\mathrm{P}<0.05$ versus transudate; ", comparison of TPE with non-TPE; ', $\mathrm{P}<0.05$ versus MPE. L/N ratio means lymphocyte to neutrophil ratio. TPE, tuberculous pleural effusion; MPE, malignant pleural effusion; IQR, interquartile range; CVA, cerebrovascular accident; COPD, chronic obstructive pulmonary disease; PE, pleural effusion; LDH, lactate dehydrogenase; CRP, C-reactive protein; ADA, adenosine deaminase; TB, tuberculosis.

(97.9\% sensitivity and $86.6 \%$ specificity) (Figure $2 B$ ). In this three-factor model, TPE was favored if the pleural fluid analysis met at least two of the following criteria: (I) ADA $\geq 40 \mathrm{IU} / \mathrm{mL}$; (II) calpain- $1 \geq 787 \mathrm{ng} / \mathrm{mL}$; and (III) SBDP $\geq 2.745 \mathrm{ng} / \mathrm{mL}$. In terms of $\mathrm{ADA} \geq 40 \mathrm{IU} / \mathrm{mL}$, the threefactor model showed increments in the sensitivity and a mild reduction in the specificity (Table 3).

The predicted probability was $>0.326$ in five MPEs (false positives). Among them, three fulfilled the calpain-1 and SBDP criteria, one fulfilled the calpain-1 and ADA criteria, and one fulfilled the SBDP and ADA criteria. In two patients, MPE was correctly diagnosed based on 

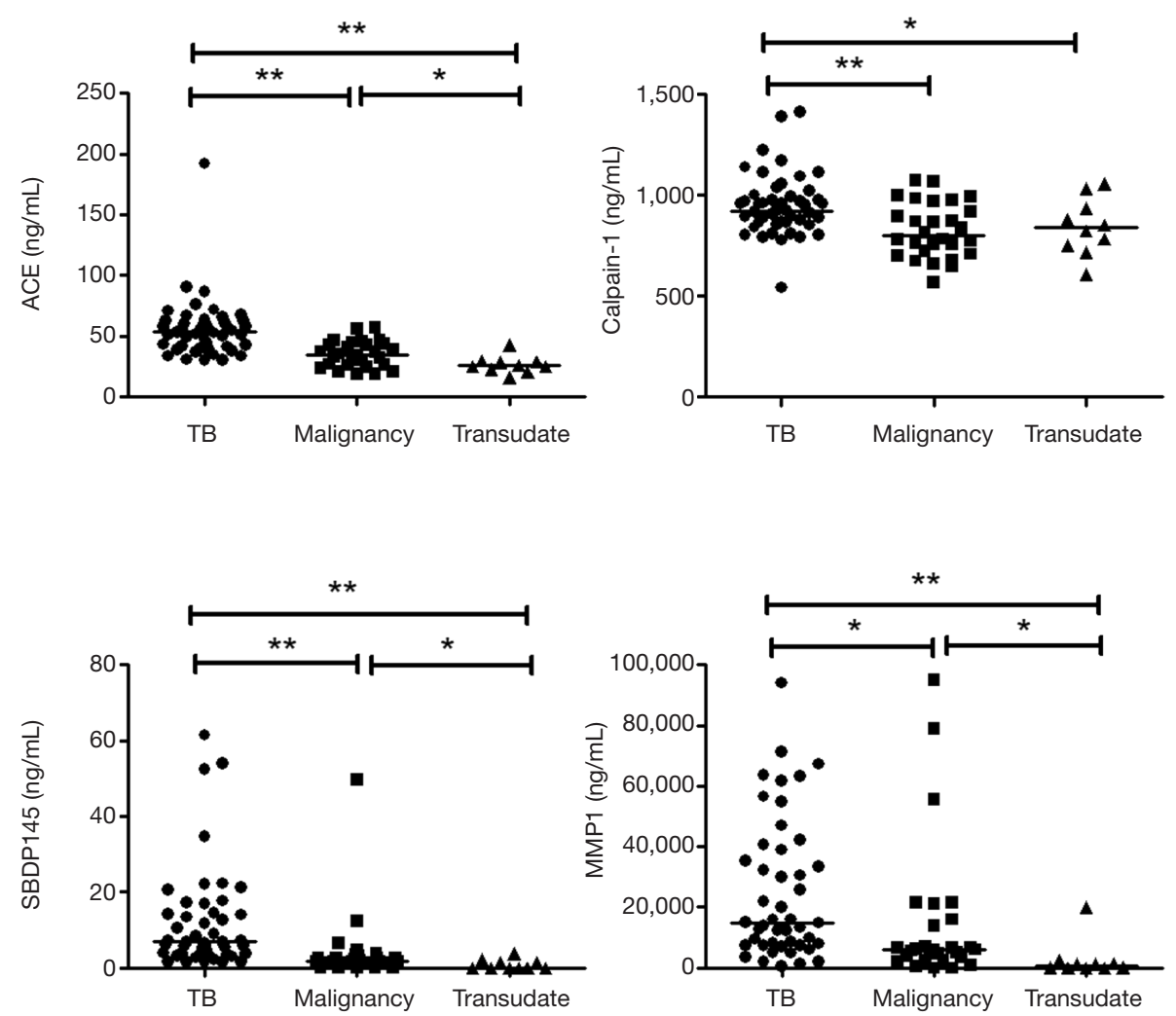

Figure 1 ACE, calpain-1, SBDP, and MMP-1 levels in pleural effusion. ** $\mathrm{P}<0.001,{ }^{*} \mathrm{P}<0.05$. ACE, angiotensin-converting enzyme; SBDP, spectrin breakdown products; MMP-1, matrix metalloproteinase-1.
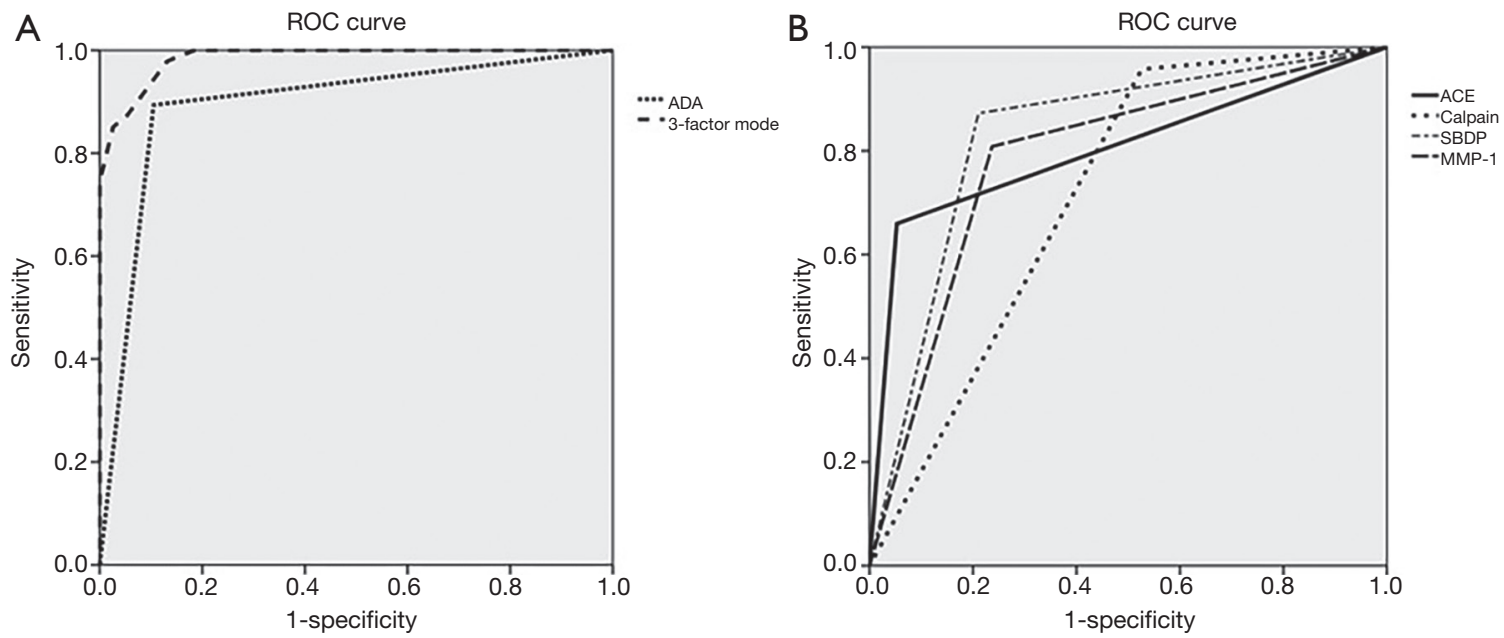

Figure 2 Receiver-operating characteristic (ROC) curves showing the accuracy of several biomarkers in the diagnosis of tuberculous pleural effusion. (A) ROC curve for ADA and the three-factor model with 95\% confidence. (B) ROC curve for ACE, calpain-1, SBDP, and MMP1 with 95\% confidence. ADA, adenosine deaminase; ACE, angiotensin-converting enzyme; SBDP, spectrin breakdown products; MMP-1, matrix metalloproteinase-1. 
Table 2 Markers of pleural fluid associated with tuberculous pleural effusion (TPE), identified through multivariate analysis

\begin{tabular}{|c|c|c|c|c|c|c|}
\hline Characteristics & Category & Patient No. & TPE (\%) & Univariate $\mathrm{P}$ value & Multivariate $P$ value & Multivariate OR $(95 \% \mathrm{Cl})$ \\
\hline ADA & $<40 \mathrm{IU} / \mathrm{mL}$ & 46 & $42(91.3)$ & & & \\
\hline \multirow[t]{2}{*}{ ACE } & $\geq 47.16 \mathrm{ng} / \mathrm{mL}$ & 52 & $16(30.8)$ & $<0.001$ & 0.107 & $24.8(0.5-1,235.5)$ \\
\hline & $<47.16 \mathrm{ng} / \mathrm{mL}$ & 33 & 31 (93.9) & & & \\
\hline Calpain-1 & $<787 \mathrm{ng} / \mathrm{mL}$ & 65 & $45(69.2)$ & & & \\
\hline \multirow[t]{2}{*}{ SBDP } & $\geq 2.745 \mathrm{ng} / \mathrm{mL}$ & 36 & $6(16.7)$ & $<0.001$ & 0.034 & $38.3(1.3-1,112.4)$ \\
\hline & $<2.745 \mathrm{ng} / \mathrm{mL}$ & 49 & $41(83.7)$ & & & \\
\hline MMP-1 & $\geq 7,229 \mathrm{pg} / \mathrm{mL}$ & 38 & $9(23.7)$ & $<0.001$ & 0.379 & $4.6(0.2-138.6)$ \\
\hline
\end{tabular}

Multivariate logistic analysis adjusted for sex, age, ADA, ACE, calpain-1, SBDP, and MMP-1. ADA, adenosine deaminase; ACE, angiotensin-converting enzyme; SBDP, spectrin breakdown products; MMP-1, matrix metalloproteinase-1; OR, odds ratio; Cl, confidence interval.

Table 3 Performance of different pleural markers for predicting tuberculous pleural effusion

\begin{tabular}{|c|c|c|c|c|c|c|}
\hline Biomarkers & Sensitivity (\%) & Specificity (\%) & PPV (\%) & NPV (\%) & LR+ & LR- \\
\hline Calpain-1 $\geq 787 \mathrm{ng} / \mathrm{mL}$ & 95.7 & 47.3 & 69.2 & 90.0 & 22.5 & 0.08 \\
\hline $\mathrm{SBDP} \geq 2.745 \mathrm{ng} / \mathrm{mL}$ & 87.2 & 78.9 & 83.6 & 83.3 & 6.8 & 0.16 \\
\hline MMP-1 $\geq 7,229 \mathrm{pg} / \mathrm{mL}$ & 74.4 & 65.7 & 72.9 & 67.5 & 2.9 & 0.38 \\
\hline Three-factor model & 97.9 & 86.8 & 88.1 & 97.6 & 7.42 & 0.02 \\
\hline
\end{tabular}

ADA, adenosine deaminase; ACE, angiotensin-converting enzyme; SBDP, spectrin breakdown products; MMP-1, matrix metalloproteinase-1; PPV, positive predictive value; NPV, negative predictive value; LR+, likelihood ratio-positive; LR-, likelihood ratio-negative.

negative results for calpain-1 and SBDP, even though the ADA was greater than 40 . On the other hand, there was one false-negative patient in this predictive model. In four patients, TPE was correctly diagnosed based on positive results for calpain-1 and SBDP, even though the ADA was less than 40.

Table 4 shows the factors associated with RPT. There was no relationship between RPT and LDH, glucose, protein, cell, or ADA in the pleural fluid at the initial thoracentesis. We found that patients with RPT had significantly higher calpain-1, SBDP, and MMP-1 levels (all, $\mathrm{P}<0.05$ ) than those without RPT.

\section{Discussion}

Markers for rapid and accurate differential diagnosis are still required for patients with PE because of the limitations of conventional diagnostic markers. Considerable numbers of patients with tuberculous pleurisy have RPT despite successfully completing treatment (11). In tuberculous pleurisy, irreversible pleural fibrosis sometimes occurs, leading to dyspnea and chronic respiratory distress. Pleural fibrosis is defined as excessive deposition and remodeling of the ECM after diverse inflammatory conditions (12). Several previous studies evaluated biomarkers related to the metabolism of the ECM protein in the pathogenesis of 
Table 4 Pleural fibrosis characteristics according to residual pulmonary fibrosis in tuberculous pleural effusion

\begin{tabular}{lccc}
\hline Pleural fluid characteristics & Residual pulmonary thickening $(+),(\mathrm{n}=19)$ & Residual pulmonary thickening $(-),(\mathrm{n}=28)$ & $\mathrm{P}$ value \\
\hline ACE $(\mathrm{ng} / \mathrm{mL})$ & $52.89(42.50-67.75)$ & $43.98(37.06-57.88)$ & 0.1063 \\
Calpain-1 $(\mathrm{ng} / \mathrm{mL})$ & $968.9(876.6-1,137.0)$ & $901(854.2-966.4)$ & 0.0228 \\
SBDP $(\mathrm{ng} / \mathrm{mL})$ & $12.62(6.05-20.55)$ & $5.605(2.858-11.510)$ & 0.0395 \\
MMP-1 $(\mathrm{pg} / \mathrm{mL})$ & $35,470[8,351-54,780]$ & $12,910[5,308-21,570]$ & 0.0294 \\
LDH & $472[359-638]$ & $504(299.3-724.8)$ & 0.745 \\
Glucose & $97[84-116]$ & $100(62.0-126.5)$ & $7.5(7.5-7.5)$ \\
pH & $7.5(7.5-8.0)$ & $4.8(4.5-5.3)$ & 0.9913 \\
Protein & $4.9(4.4-5.6)$ & $1,440(615.8-2,891.0)$ \\
Cells & $1,152[864-1,872]$ & $97(68.8-120.0)$ \\
ADA & $98.6(79.7-107.0)$ & 0.6879 \\
\hline
\end{tabular}

ADA, adenosine deaminase; ACE, angiotensin-converting enzyme; SBDP, spectrin breakdown products; MMP-1, matrix metalloproteinase-1; LDH, lactate dehydrogenase.

fibrosis (6,7,13-19). The diagnostic application of markers associated with fibrosis and remodeling are worthy of attention.

ACE has been shown to be a key mediator of the pathogenesis of fibrotic diseases, such as idiopathic pulmonary fibrosis and cardiovascular fibrosis $(13,14)$. A number of studies indicated that the ACE-Ang II axis modulates the metabolism of the ECM protein, in addition to other relevant protein activity $(15,16)$.

Soderblom et al. found that measuring ACE in PEs may aid in differentiating rheumatoid and tuberculous pleurisy from other pleural diseases (20). Hsieh et al. reported that the ACE/ACE2 ratio and MMP-9 activity in PE may be potential biomarkers for diagnosing tuberculous pleurisy (15).

Calpain is a family of calcium-dependent endopeptidases that plays an important role in ECM remodeling (17). A previous study found that calpain-1 was a novel therapeutic target to retard age-associated ECM remodeling in hypertension and atherosclerosis (17). Calpain-mediated cleavage of the submembrane cytoskeleton is important in lens cell differentiation (21). Yang et al. reported that in tuberculous pleural fibrosis, calpain activation by the ACEAng II axis plays an important role, and that the calpain inhibitor MDL28170 reduced collagen production (6).

SBDP comprises molecules released after cleavage by calpain (22). SBDPs are novel biomarkers for aging and acute neuronal damage, such as traumatic brain injury (23).

Some studies showed that MMP-1 in pleural fluid is increased in tuberculous pleuritis in comparison to other etiologies $(18,19)$. MMP-1 contributes to fibrotic reactions by cleaving the ECM and driving lung matrix destruction without caseous necrosis (19).

To the best of our knowledge, this is the first study to simultaneously explore the markers associated with pleural fibrosis, such as ACE, calpain-1, SBDP, and MMP-1, in $\mathrm{PE}$. Our results are in agreement with the hypotheses of previous studies.

In the present study, patients with TPE had higher levels of ACE, calpain-1, SBDP, and MMP-1 in PE versus those with MPE and transudate. This difference in the expression of ACE, calpain-1, SBDP, and MMP-1 between TPE and non-TPE groups may reflect a gap in the disease pathogenesis. On multivariate logistic regression, ADA $\geq 40 \mathrm{IU} / \mathrm{mL}$, calpain- $1 \geq 787 \mathrm{ng} / \mathrm{mL}$, and SBDP $\geq 2.745 \mathrm{ng} / \mathrm{mL}$ were independent predictors of TPE. If the probability predicted by the regression model is $\geq 0.326$ ( $97.9 \%$ sensitivity and $86.6 \%$ specificity), TPE is favored when the $\mathrm{PE}$ analysis fulfills at least two out of the above three criteria (for ADA, calpain-1, and SBDP).

$\mathrm{ADA}$ is known to be the most reliable biomarker of $\mathrm{TB}$ pleurisy (24). However, the ADA level can be less sensitive in immunocompromised patients when using a cut-off value of $40 \mathrm{IU} / \mathrm{mL}$ (25). Moreover, an elevated pleural fluid ADA level can also be seen in other diseases, such as empyema and lymphoma (26). By simultaneously measuring calpain-1 and SBDP, the three-factor predictive model improved the sensitivity compared with ADA alone, and continued to 
show high sensitivity.

Our result showing that the glucose or LDH levels of pleural fluid do not depend on residual pleural fibrosis coincides with the results of Barbas et al. (9). Residual pleural fibrosis is related to higher levels of calpain-1, SBDP, and MMP-1 among TPE cases. It would be of interest in future studies to verify whether these markers play a role as monitoring markers for the prediction fibrosis and prognosis.

The present study had some limitations. First, the number of participants was small. Further studies are needed to validate our findings in a larger cohort. Second, this study did not consider the health cost when novel biomarkers are added to ADA in clinical practice. Third, there were no follow-up data for these biomarkers to allow assessment of the prognostic clinical value.

In conclusion, we demonstrated that ACE, calpain-1, SBDP, and MMP-1 are fibrosis-associated biomarkers that can be applied to differentiate TPE from non-TPE. Measuring pleural ADA, calpain-1, and SBDP together may improve the diagnostic efficacy for TPE compared with assaying $\mathrm{ADA}$ alone.

In TPE, high pleural levels of calpain-1, SBDP, and MMP-1 may indicate residual pleural fibrosis. Further studies are needed to determine the clinical value of calpain-1 and SBDP with respect to diagnostic and therapeutic aspects in TPE.

\section{Acknowledgements}

Funding: This work was supported by the Hallym University Research Fund (HURF-2016-20). This research was supported by the Bio \& Medical Technology Development Program of the National Research Foundation (NRF) \& funded by the Korean government (MSIT) (NRF2017M3A9E8033225) and was supported by the National Research Foundation of Korea Grant funded by Korean Government (NRF 2017R1C1B5017879).

\section{Footnote}

Conflicts of Interest: The authors have no conflicts of interest to declare.

Ethical Statement: The study was approved by institutional ethics board of Chuncheon Sacred Hospital (No. 2012-27). Written informed consent was obtained from the patient for publication of this manuscript and any accompanying images.

\section{References}

1. Sharma SK, Mohan A. Extrapulmonary tuberculosis. Indian J Med Res 2004;120:316-53.

2. Conde MB, Loivos AC, Rezende VM, et al. Yield of sputum induction in the diagnosis of pleural tuberculosis. Am J Respir Crit Care Med 2003;167:723-5.

3. de Pablo A, Villena V, Echave-Sustaeta J, et al. Are pleural fluid parameters related to the development of residual pleural thickening in tuberculosis? Chest 1997;112:1293-7.

4. Ma W, Han W, Greer PA, et al. Calpain mediates pulmonary vascular remodeling in rodent models of pulmonary hypertension, and its inhibition attenuates pathologic features of disease. J Clin Invest 2011;121:4548-66.

5. Tabata C, Tabata R, Nakano T. The calpain inhibitor calpeptin prevents bleomycin-induced pulmonary fibrosis in mice. Clin Exp Immunol 2010;162:560-7.

6. Yang J, Xiang F, Cai PC, et al. Activation of calpain by renin-angiotensin system in pleural mesothelial cells mediates tuberculous pleural fibrosis. Am J Physiol Lung Cell Mol Physiol 2016;311:L145-53.

7. Chen WL, Sheu JR, Chen RJ, et al. Mycobacterium tuberculosis Upregulates TNF-alpha Expression via TLR2/ERK Signaling and Induces MMP-1 and MMP9 Production in Human Pleural Mesothelial Cells. PLoS One 2015;10:e0137979.

8. Light RW, Macgregor MI, Luchsinger PC, et al. Pleural effusions: the diagnostic separation of transudates and exudates. Ann Intern Med 1972;77:507-13.

9. Barbas CS, Cukier A, de Varvalho CR, et al. The relationship between pleural fluid findings and the development of pleural thickening in patients with pleural tuberculosis. Chest 1991;100:1264-7.

10. Burgess LJ, Maritz FJ, Le Roux I, et al. Combined use of pleural adenosine deaminase with lymphocyte/neutrophil ratio. Increased specificity for the diagnosis of tuberculous pleuritis. Chest 1996;109:414-9.

11. Chan CH, Arnold M, Chan CY, et al. Clinical and pathological features of tuberculous pleural effusion and its long-term consequences. Respiration 1991;58:171-5.

12. Huggins JT, Sahn SA. Causes and management of pleural fibrosis. Respirology 2004;9:441-7.

13. Uh ST, Kim TH, Shim EY, et al. Angiotensin-converting enzyme (ACE) gene polymorphisms are associated with idiopathic pulmonary fibrosis. Lung 2013;191:345-51.

14. Varagic J, Ahmad S, Voncannon JL, et al. Nebivolol reduces cardiac angiotensin II, associated oxidative 
stress and fibrosis but not arterial pressure in saltloaded spontaneously hypertensive rats. J Hypertens 2012;30:1766-74.

15. Hsieh WY, Kuan TC, Cheng KS, et al. ACE/ACE2 ratio and MMP-9 activity as potential biomarkers in tuberculous pleural effusions. Int J Biol Sci 2012;8:1197-205.

16. Pan $\mathrm{CH}$, Wen CH, Lin CS. Interplay of angiotensin II and angiotensin(1-7) in the regulation of matrix metalloproteinases of human cardiocytes. Exp Physiol 2008;93:599-612.

17. Jiang L, Zhang J, Monticone RE, et al. Calpain-1 regulation of matrix metalloproteinase 2 activity in vascular smooth muscle cells facilitates age-associated aortic wall calcification and fibrosis. Hypertension 2012;60:1192-9.

18. Hoheisel G, Sack U, Hui DS, et al. Occurrence of matrix metalloproteinases and tissue inhibitors of metalloproteinases in tuberculous pleuritis. Tuberculosis (Edinb) 2001;81:203-9.

19. Sundararajan S, Babu S, Das SD. Comparison of localized versus systemic levels of Matrix metalloproteinases (MMPs), its tissue inhibitors (TIMPs) and cytokines in tuberculous and non-tuberculous pleuritis patients. Hum Immunol 2012;73:985-91.

20. Soderblom T, Nyberg P, Pettersson T, et al. Pleural fluid beta-2-microglobulin and angiotensin-converting enzyme concentrations in rheumatoid arthritis and tuberculosis. Respiration 1996;63:272-6.

21. De Maria A, Shi Y, Kumar NM, et al. Calpain expression and activity during lens fiber cell differentiation. J Biol Chem 2009;284:13542-50.

22. Pike BR, Flint J, Dave JR, et al. Accumulation of calpain and caspase- 3 proteolytic fragments of brain-derived alphaII-spectrin in cerebral spinal fluid after middle cerebral artery occlusion in rats. J Cereb Blood Flow Metab 2004;24:98-106.

23. Yan XX, Jeromin A, Jeromin A. Spectrin Breakdown Products (SBDPs) as Potential Biomarkers for Neurodegenerative Diseases. Curr Transl Geriatr Exp Gerontol Rep 2012;1:85-93.

24. Porcel JM. Tuberculous pleural effusion. Lung 2009;187:263-70.

25. Valdes L, Alvarez D, San Jose E, et al. Tuberculous pleurisy: a study of 254 patients. Arch Intern Med 1998;158:2017-21.

26. Valdes L, Alvarez D, Valle JM, et al. The etiology of pleural effusions in an area with high incidence of tuberculosis. Chest 1996;109:158-62.
Cite this article as: Hong JY, Park SY, Kim Y, Lee CY, Lee MG. Calpain and spectrin breakdown products as potential biomarkers in tuberculous pleural effusion. J Thorac Dis 2018;10(5):2558-2566. doi: 10.21037/jtd.2018.04.85 